\title{
Nanoporous Gold Surface: An Efficient Platform for Hydrogen Evolution Reaction at Very Low Overpotential
}

\author{
Anandhakumar Sukeri and Mauro Bertotti* \\ Departamento de Química Fundamental, Instituto de Química, Universidade de São Paulo, \\ Av. Professor Lineu Prestes, 748, 05513-970 São Paulo-SP, Brazil
}

\begin{abstract}
A nanostructured porous gold film (NPGF) was fabricated onto a gold electrode surface by an electrochemical approach involving anodization followed by electrochemical reduction in absence of binary alloys, templates and chemical reducing agents. The electrocatalytic activity of the as-fabricated electrocatalyst was examined towards hydrogen evolution reaction (HER) and very low onset potential $(-0.048 \mathrm{~V})$ and overpotential values of -0.070 and $-0.083 \mathrm{~V}$ at current densities of 10 and $20 \mathrm{~mA} \mathrm{~cm}^{-2}$, respectively, were obtained in $0.5 \mathrm{~mol} \mathrm{~L}^{-1}$ sulfuric acid solution, along with exchange current density $\left(\mathrm{j}_{\mathrm{o}}\right.$ ) of $0.126 \mathrm{~mA} \mathrm{~cm}^{-2}$. A small Tafel slope of ca. $36 \mathrm{mV}$ decade ${ }^{-1}$ was achieved, indicating the HER takes place according to a Volmer-Heyrovsky mechanism and that the electrochemical desorption of hydrogen is the rate determining step. The performance of the NPGF electrode was compared with that of bulk gold and platinum electrodes, and a platinum-like behavior was noticed for HER. Finally, the durability of the proposed electrocatalyst was examined using voltammetric and amperometric techniques and an excellent stability was achieved.
\end{abstract}

Keywords: nanostructured porous gold, electrocatalyst, hydrogen evolution reaction, platinumlike performance

\section{Introduction}

Most electricity generation methods produce $\mathrm{CO}_{2}$ and such environmental impact can be minimized by using renewable processes. ${ }^{1,2}$ In this context, hydrogen is considered to be one of the most important fuels for future energy applications due to its high energy density and the demand for clean and renewable energy sources. ${ }^{3,4}$ The main existing process for hydrogen production is based on steam reforming of methane, but such reaction requires catalysts and high energy as input, and $\mathrm{CO}_{2}$ is as a by-product. ${ }^{5,6}$ Therefore, it is essential to find alternative ways to produce $\mathrm{CO}_{2}$-free hydrogen at room temperature.

Water splitting or HER (hydrogen evolution reaction) is an important pathway to produce hydrogen with high purity, as this approach allows to store energy without the emission of $\mathrm{CO}_{2}$. Accordingly, the development of simple methods to fabricate efficient electrocatalysts for HER is highly desirable, even though the limiting step in water splitting and water electrolysis is the oxygen evolution reaction. However, the synthesis of such kind of catalysts (that minimize the activation energy for hydrogen formation on the electrode

*e-mail: mbertott@iq.usp.br surface) still remains a challenge. ${ }^{7}$ It is well-known that platinum and its alloys are the benchmark electrocatalysts for HER because of the low overpotential and fast kinetics required for driving the reaction. ${ }^{8-10}$ Unfortunately, the limited supply and expensiveness of these metals make them unattractive. As a result, enormous efforts have been devoted to finding alternative catalysts for producing hydrogen at low overpotential. For example, cobalt, ${ }^{11-13}$ nickel, ${ }^{14}$ copper $^{15-17}$ bimetallic catalyst,$^{18}$ transition metal carbide nanocrystals M3C (M: Fe, Co, Ni) encapsulated with vertically aligned graphene nanoribbons, ${ }^{19}$ nitrogen and sulfur co-doped nanoporous graphene, ${ }^{20}$ molybdenum sulfide $\left(\mathrm{MoS}_{2}\right)$, phosphide, carbon and noble metal-free catalysts are reported for the HER process. ${ }^{21-24}$

Currently, nanoporous gold films (NPGF) have attracted attention for their widespread application in catalysis and in the sensors field because of their higher surface area in comparison to bulk gold electrodes, allowing superior electron transfer rate between the electrode-electrolyte interface. ${ }^{25,26}$ Such platforms have been mainly prepared by alloying/dealloying or template methods, where hazardous chemicals are mostly used. ${ }^{27,28}$ Hence, it is really a challenging task to develop convenient and green methods to fabricate NPGF with high surface area. Gold surfaces 
with such features have been produced by square wave potential perturbations, ${ }^{29}$ anodization in oxalate solution, ${ }^{30}$ anodization of gold in $\mathrm{HCl}$ medium, ${ }^{31}$ dealloying of silver/gold alloys ${ }^{32}$ anodization using ascorbic acid as reducing agents ${ }^{33}$ and electroless deposition of iridium oxide nanoparticles on an anodized gold surface. ${ }^{34}$ Also, gold nanomaterials have been found to be catalytically active and relatively stable in acid medium. ${ }^{35}$ Hitherto, HER activity has been studied on gold single crystal electrodes, ${ }^{36}$ electrochemically synthesized gold nanospikes and electrochemically grown mesoporous gold thin film. ${ }^{37,38}$ Moreover, nanoporous gold/tungsten sulfide composite film, ${ }^{39}$ and Au-CNT and Au-graphene composite ${ }^{35}$ have been used as electrocatalysts for HER.

Recently, the fabrication of a high surface area nanoporous gold film electrode via anodization followed by electrochemical reduction in a sulfuric acid medium for dissolved oxygen reduction was proposed for the first time by our research group. ${ }^{40}$ In this work, the attempt has been made to evaluate the electrocatalytic activity of the NPGF electrode towards HER in acid medium and excitingly we observed a platinum-like performance at very low overpotential along with excellent durability.

\section{Experimental}

\section{Reagents}

Sulfuric acid (Merck) was used as received. A $0.5 \mathrm{~mol} \mathrm{~L}^{-1} \mathrm{H}_{2} \mathrm{SO}_{4}$ solution was prepared and served as an electrolytic solution for the fabrication of the NPGF electrode and in hydrogen evolution reaction studies. A high pure Milli-Q-water (18 M $\Omega \mathrm{cm}$ ) was used for all necessary solutions preparation.

\section{Apparatus}

The electrochemical experiments were carried out using a PalmSens portable potentiostat (BV, Netherland) using a three-electrode electrochemical system: the electrochemically fabricated NPGF electrode, a platinum foil and $\mathrm{Ag} / \mathrm{AgCl}$ (saturated $\mathrm{KCl}$ ) served as working, counter and reference electrodes, respectively. All potential values reported here were converted to reversible hydrogen electrode (RHE) and all the polarization curves were iR-corrected. Current density values $\left(\mathrm{j}\right.$, in $\mathrm{mA} \mathrm{cm}^{-2}$ ) used in this work were calculated from the geometrical area of the Au electrode $\left(0.07065 \mathrm{~cm}^{2}\right){ }^{35}$ A JOEL JSM740F equipment with an acceleration voltage of $30 \mathrm{kV}$ was used to take the FE-SEM (field emission scanning electron microscopy) images.
Fabrication of the NPGF electrode

The nanoporous gold film electrode was fabricated using our recent approach. ${ }^{40}$ Briefly, the potential of a gold electrode was cycled from 0.0 to $2.0 \mathrm{~V}$ at the scan rate of $0.02 \mathrm{~V} \mathrm{~s}^{-1}$ in a $0.5 \mathrm{~mol} \mathrm{~L}^{-1} \mathrm{H}_{2} \mathrm{SO}_{4}$ solution and the electrode potential was held at $2.0 \mathrm{~V}$ for $60 \mathrm{~min}$ after the forward scan, where a gold oxide film (orange-yellowish color) was formed on the electrode surface. Afterwards, the generated oxide film was reduced in the reverse scan to form a nanoporous gold film (black color).

\section{Results and Discussion}

\section{Microscopic analysis of NPGF surface}

FE-SEM images of bare $\mathrm{Au}$ and electrochemically fabricated NPGF electrodes are shown in Figures 1A and $1 \mathrm{~B}$, respectively. A smooth surface with slight scratches are observed for the bare Au electrode, whereas an extremely rough surface with porous nanostructures with an average pore size of $32 \mathrm{~nm}$ along with large defective sites and/or edges was noticed for the NPGF electrode. ${ }^{40}$ Typically, such kind of pore structure has large surface area and roughness factor and can be more beneficial for electron transfer processes, leading to enhanced electrocatalytic activity for many electrochemical reactions. Besides, recently we witnessed the absence of oxide formation on the fabricated NPGF surface through energy dispersive X-ray analysis (EDX) ${ }^{41}$ which confirms the outstanding purity of the NPGF compared to the material prepared by dealloying or template based methods.

\section{Electrochemical characterization of the NPGF electrode}

The cyclic voltammetric responses of bare $\mathrm{Au}$ and NPGF electrodes in $0.5 \mathrm{~mol} \mathrm{~L}^{-1} \mathrm{H}_{2} \mathrm{SO}_{4}$ solution at the scan rate of $0.1 \mathrm{~V} \mathrm{~s}^{-1}$ are shown in Figure 2. A large current signal was observed at the peak potential of $0.9 \mathrm{~V}$ for the NPGF electrode, corresponding to the reduction of $\mathrm{Au}$ oxides formed in the forward scan. Such process is much more pronounced in comparison to the corresponding one at the polycrystalline Au electrode. This can be reasoned as a consequence of the evolution of oxygen gas bubbles at the electrode surface during the anodization process, hence a porous structure with increased surface area is created. Also, in order to further confirm the porous nature of the $\mathrm{Au}$ film, the electrochemical surface area (ECSA) was calculated using the Au oxide reduction peak by a charge-integration method. ${ }^{40}$ Values were found to be 0.34 and $5.12 \mathrm{~cm}^{2}$ for bare $\mathrm{Au}$ and the NPGF electrode, 

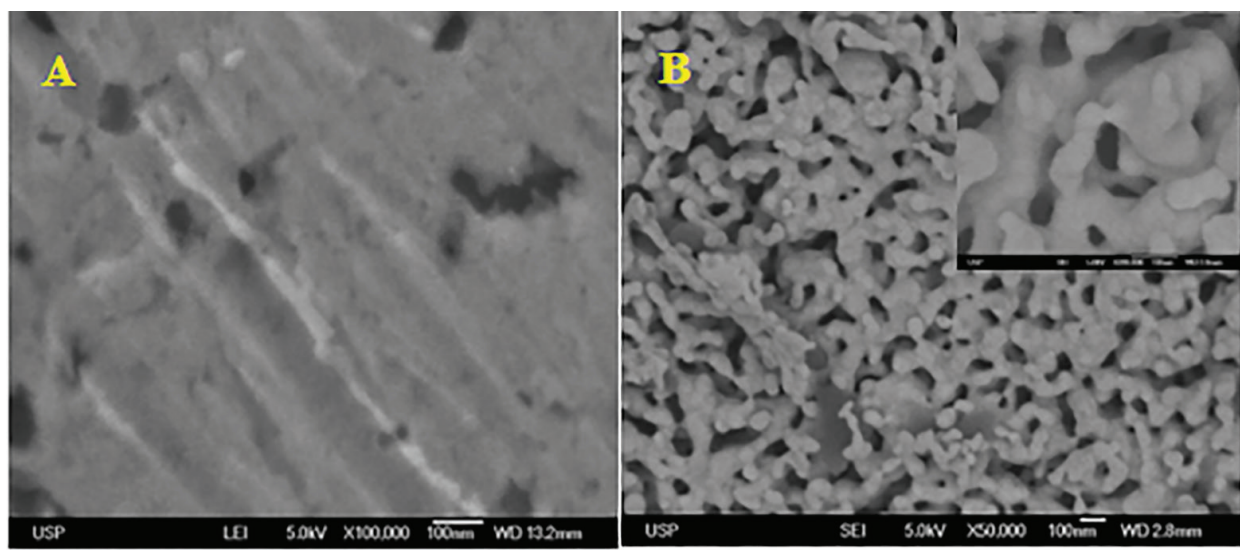

Figure 1. FE-SEM images of bare Au (A) and NPGF electrode (B).

respectively. Additionally, surface roughness factor (RF, i.e. ratio between the ECSA and geometrical area of the electrode) was also calculated and the values 4.81 and 72.47 were obtained for both electrodes, respectively. Such values indicated an approximately 15 -fold enhancement of ECSA and RF by comparing the fabricated NPGF electrode with the bare Au electrode.

Jeyabharathi et $a l .{ }^{42}$ have recently shown the relationship between the Au oxide peaks and the low-index crystal planes of the polycrystalline gold by using cyclic voltammetry in acid medium. The polycrystalline gold surface showed a single oxidation peak due to the amorphous nature of the surface, whereas after repetitive potential cycling three oxidation peaks emerged at the peak potentials of 1.14, 1.20 and $1.35 \mathrm{~V}$. Similar peaks in the voltammogram recorded with the NPGF electrode can be noticed in Figure 2. This was attributed by the authors to the formation of (i) Au oxides, (ii) surface roughness and (iii) defects in the crystal structure, which are related to the low index crystal planes of $\mathrm{Au}(100), \mathrm{Au}(110)$ and $\mathrm{Au}(111)$, respectively. Hence, it is likely that our procedure involving the anodization of the Au surface followed by electrochemical reduction strongly influences the structure of the $\mathrm{Au}$ electrode surface in a similar pathway, i.e., low-index crystal planes are generated. At such active sites, reactants can be more easily adsorbed. As a consequence, reactants are able to participate in inner-sphere electron transfer reactions and the rate of such processes is enhanced.

\section{Electrocatalytic activity of the NPGF electrode for HER}

The catalytic activity of the electrochemically fabricated NPGF electrode for HER was explored in a $0.5 \mathrm{~mol} \mathrm{~L}^{-1} \mathrm{H}_{2} \mathrm{SO}_{4}$ solution. Prior to each measurement, dissolved oxygen was removed by purging argon gas into the electrolytic solution. In order to compare the electrochemical activity, experiments were also

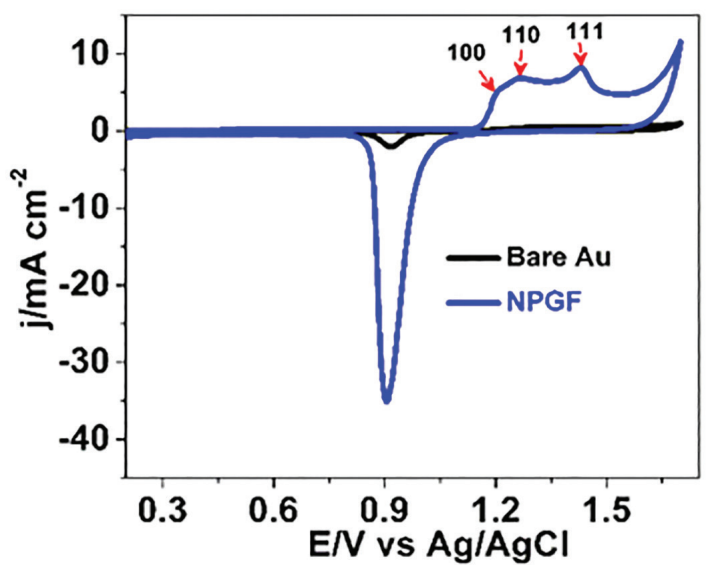

Figure 2. Cyclic voltammetric responses of bare Au and NPGF electrode in $0.5 \mathrm{~mol} \mathrm{~L}^{-1} \mathrm{H}_{2} \mathrm{SO}_{4}$ solution. Scan rate $0.1 \mathrm{~V} \mathrm{~s}^{-1}$.

performed using bare gold and platinum electrodes (same geometrical area). Polarization curves were obtained using linear sweep voltammetry at the scan rate of $2 \mathrm{mV} \mathrm{s}^{-1}$ (Figure 3). As it can be seen, when the holding time increased from 10 to $60 \mathrm{~min}$, the overpotential for HER was shifted towards less negative potentials (we did not observe any change in the activity after $60 \mathrm{~min}$ (Figure S1, Supplementary Information), hence the deposition time selected as optimum for the fabrication of NPGF was $60 \mathrm{~min}$ ), whereas a poor HER activity was exhibited when a bare gold electrode was used. Additionally, very small overpotential values of $-0.070 \mathrm{~V}$ at $10 \mathrm{~mA} \mathrm{~cm} \mathrm{~cm}^{-2}$ and $-0.083 \mathrm{~V}$ at $20 \mathrm{~mA} \mathrm{~cm}{ }^{-2}$ were found for the NPGF electrode fabricated by using a 60 min holding time. Beyond those overpotentials, the cathodic current rapidly increased at more negative potentials. This performance is very close to the one of a platinum electrode and this is attributable to the fact that the NPGF has more active sites (high surface area and/or high roughness factor), resulting in superior electrocatalytic activity for the HER process.

The mechanism of hydrogen evolution reaction in acid medium has been evaluated based on the slope value 


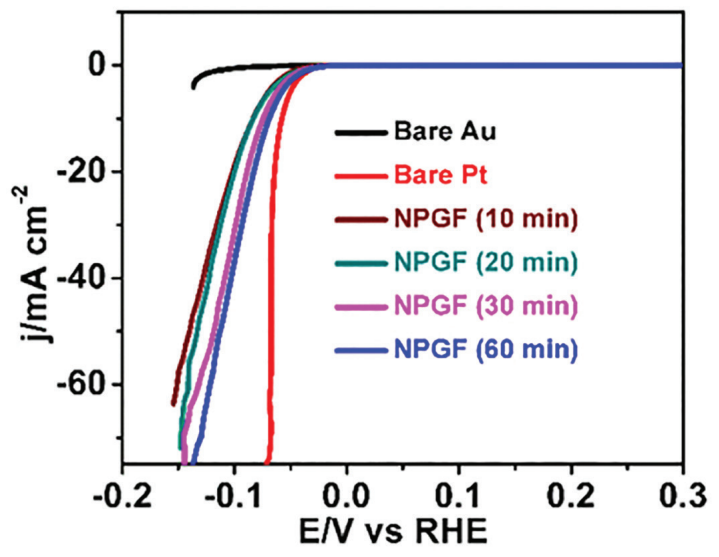

Figure 3. Linear sweep voltammograms of bare $\mathrm{Au}$, Pt and NPGF electrodes in $0.5 \mathrm{~mol} \mathrm{~L}^{-1} \mathrm{H}_{2} \mathrm{SO}_{4}$ solution at a scan rate of $2 \mathrm{mV} \mathrm{s}^{-1}$.

obtained from the Tafel plot (Figure 4). ${ }^{39}$ In general, HER takes places according to the following three steps:

Step 1. Primary discharge step (Volmer reaction)

$\mathrm{NPGF}+\mathrm{H}_{3} \mathrm{O}^{+}+\mathrm{e}^{-} \rightarrow$ NPGF- $\mathrm{H}_{\text {ads }}+\mathrm{H}_{2} \mathrm{O}$;

$\mathrm{b}=4.6 \mathrm{RT} / \mathrm{F} \quad \sim 120 \mathrm{mV}$

Step 2. Electrochemical desorption step (Heyrovsky reaction)

NPGF- $\mathrm{H}_{\text {ads }}+\mathrm{H}_{3} \mathrm{O}^{+}+\mathrm{e}^{-} \rightarrow \mathrm{NPGF}+\mathrm{H}_{2}+\mathrm{H}_{2} \mathrm{O}$;

$\mathrm{b}=4.6 \mathrm{RT} / 3 \mathrm{~F} \quad \sim 40 \mathrm{mV}$

Step 3. Recombination step (Tafel reaction)

NPGF-H $\mathrm{H}_{\text {ads }}+\mathrm{NPGF}-\mathrm{H}_{\text {ads }} \rightarrow \mathrm{NPGF}+\mathrm{H}_{2}$;

$\mathrm{b}=2.3 \mathrm{RT} / 2 \mathrm{~F} \quad \sim 30 \mathrm{mV}$

where $\mathrm{R}$ is the ideal gas constant, $\mathrm{T}$ is the absolute temperature, and $\mathrm{F}$ is the Faraday constant. Hence, for a metal surface, either Volmer-Heyrovsky or Volmer-Tafel are the predominant mechanisms for HER in acid medium. A $30 \mathrm{mV} \mathrm{decade}{ }^{-1}$ as Tafel slope indicates that HER takes place through the Volmer-Tafel mechanism and the recombination step is the rate determining step, whereas a Tafel slope of $40 \mathrm{mV}$ decade ${ }^{-1}$ suggests the process follows the Volmer-Heyrovsky mechanism and the electrochemical desorption step would be the rate determining step. Tafel slope values of ca. 24 and ca. $36 \mathrm{mV}$ decade ${ }^{-1}$ were obtained for platinum and NPGF electrodes, respectively. The observed Tafel slope of $36 \mathrm{mV}$ decade ${ }^{-1}$ for the NPGF electrode is much lower compared to other reported catalysts (Table S1, Supplementary Information), suggesting the electrochemical desorption step (equation 2) is the rate-determining step and therefore the HER at the NPGF electrode takes place according to the Volmer-Heyrovsky mechanism.

The onset potential of NPGF catalysed HER was obtained from the current density $v s$. potential plot

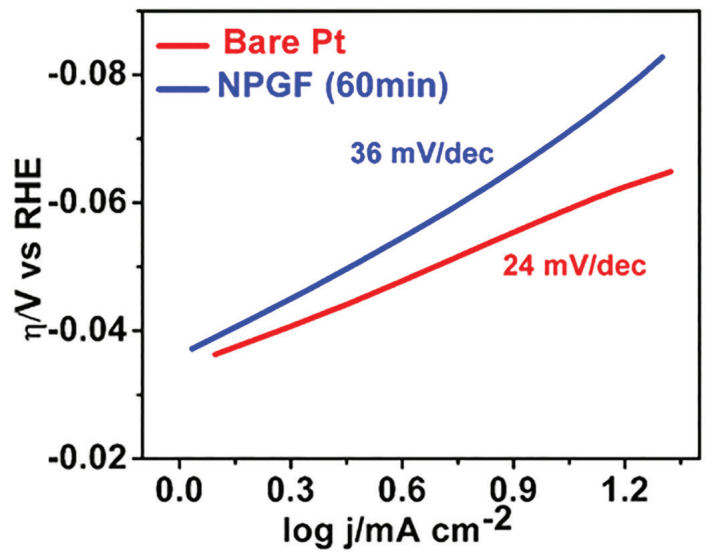

Figure 4. Tafel plot of bare Pt and NPGF electrodes (from the polarization curves of Figure 3).

(Figure 5A) and the value was determined as $-0.048 \mathrm{~V}$, which is very close to the one corresponding to a bare platinum electrode $(-0.043 \mathrm{~V})$. These results show that NPGF electrode behaves like platinum concerning HER. Furthermore, the exchange current density $\left(\mathrm{j}_{\mathrm{o}}\right)$ was calculated by the extrapolation method, i.e., $\mathrm{j}_{\mathrm{o}}$ is the current density measured at which the overpotential is $0.0 \mathrm{~V}$ in the Tafel plot. ${ }^{43}$ The obtained $\log \mathrm{j}_{\mathrm{o}}$ value was determined by extrapolation and found to be -0.9 (Figure $5 B$ ), hence $j_{0}$ was calculated as $0.126 \mathrm{~mA} \mathrm{~cm}^{-2}$. The obtained overpotential, onset potential, Tafel slope and exchange current density values are comparable to other gold (Au-CNT and Au-Graphene, and NPG/tungsten composite films) and $\mathrm{MoS}_{2}$ based catalysts and these features demonstrate the excellent electrocatalytic activity of the NPGF electrode (Table S1, Supplementary Information).

\section{Stability studies of NPGF electrode}

Another significant parameter for the evaluation of a good electrocatalyst is its stability. Accordingly, amperometric measurements were carried out at a constant potential of $-0.1 \mathrm{~V}$ for $20 \mathrm{~h}$ in a $0.5 \mathrm{~mol} \mathrm{~L}^{-1} \mathrm{H}_{2} \mathrm{SO}_{4}$ stirred solution (Figure 6). An enormous amount of hydrogen bubbles released from the electrode surface was noticed at the beginning of the experiment and the current density at the NPGF electrode decreased for ca. $1 \mathrm{~h}$. Then, a continuous and slight current increase was observed for the next $19 \mathrm{~h}$ of continuous process, but one can consider that the response did not change significantly during such monitoring, confirming the stability of the platform towards HER. Besides, linear sweep voltammograms were also recorded with the NPGF electrode in $0.5 \mathrm{~mol} \mathrm{~L}^{-1}$ $\mathrm{H}_{2} \mathrm{SO}_{4}$ solution before and after 500 consecutive cyclic voltammograms (Figure 6, inset) and both curves are superimposed, which is a clear indication of the excellent 

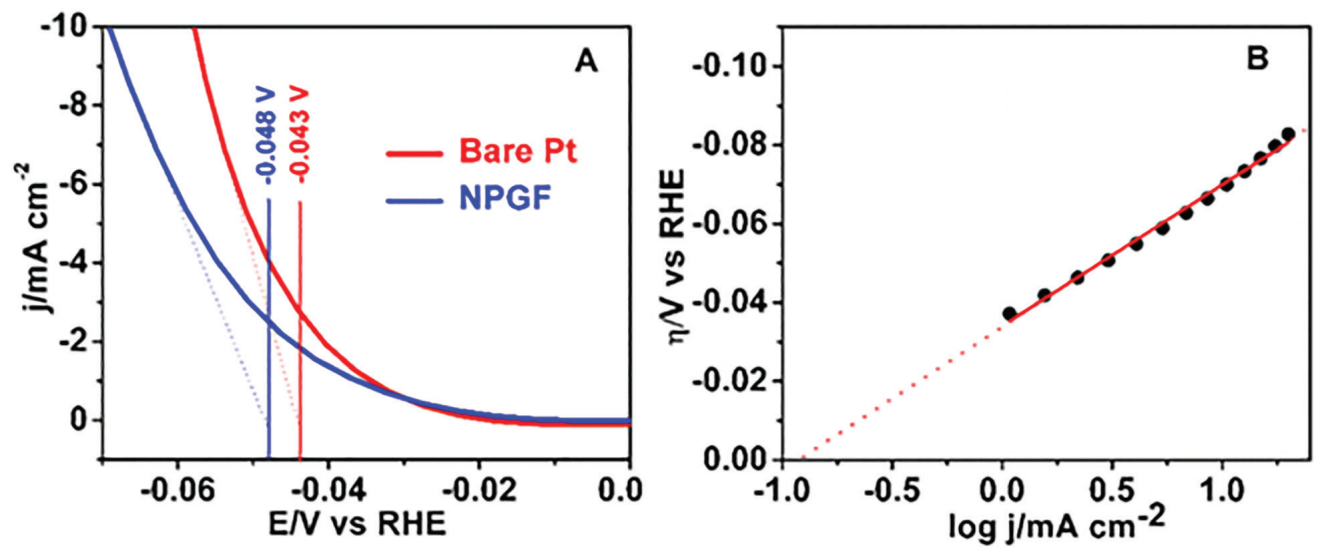

Figure 5. Potential vs. current density plot: onset potential (A); exchange current density $\left(\mathrm{j}_{\mathrm{o}}\right)(\mathrm{B})$ for HER at the NPGF electrode.

stability of the proposed platform. Hence, these results suggest the robustness and long-term durability of the developed NPGF electrocatalyst.

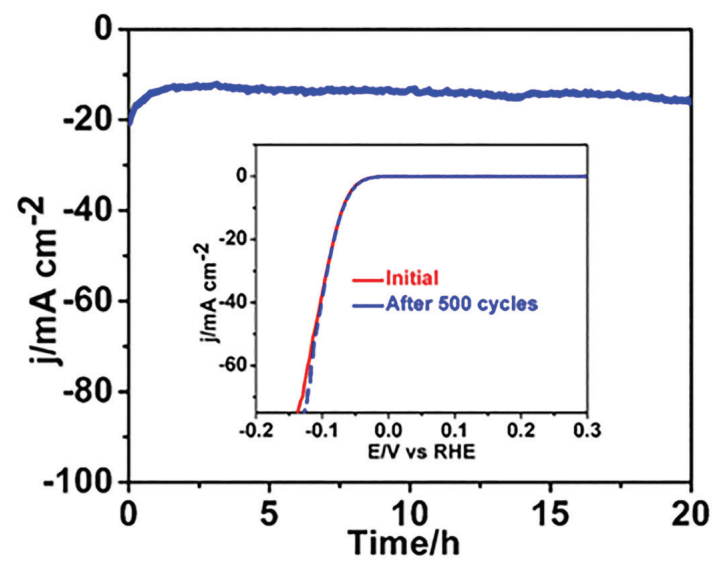

Figure 6. Amperometric response of the NPGF electrode as a function of time at $\mathrm{E}=-0.1 \mathrm{~V}$ in $0.5 \mathrm{~mol} \mathrm{~L}^{-1} \mathrm{H}_{2} \mathrm{SO}_{4}$ solution. Inset corresponds to linear sweep voltammetric responses recorded with the NPGF electrode in $0.5 \mathrm{~mol} \mathrm{~L}^{-1} \mathrm{H}_{2} \mathrm{SO}_{4}$ solution at a scan rate of $2 \mathrm{mV} \mathrm{s}^{-1}$ before and after 500 consecutive cyclic voltammograms.

\section{Conclusions}

In summary, we have examined the electrocatalytic activity of the as-prepared NPGF electrode for HER. The developed nanostructured porous gold based electrocatalyst exhibited excellent electrocatalytic activity in terms of low onset and overpotential with large cathodic current density, small Tafel slope along with excellent long-term stability. Such perfomance towards HER is similar to the one corresponding to platinum. Therefore, we believe the results shown in this work may be a starting point for the development of novel nanoporous based electrocatalysts, which can have many potential applications in the field of electrocatalysis and electrochemical (bio) sensors. As a matter of fact, the NPGF microelectrode can replace a Pt tip in scanning electrochemical microscopy (SECM) experiments involving the electroxidation of in situ generated hydrogen and studies are under progress.

\section{Supplementary Information}

The catalyst activity after 60 min of anodization and the comparison of catalytic performance with other catalysts reported in the literature are given in Figure S1 and Table S1, respectively, which are available free of charge at http://jbcs.sbq.org.br as PDF file.

\section{Acknowledgments}

Anandhakumar Sukeri thanks FAPESP for the award of Postdoctoral Research grant (2014/15215-5) and the authors also thank FAPESP (2015/20776-9), CAPES and $\mathrm{CNPq}$ for financial assistance. Furthermore, Anandhakumar Sukeri thanks Dr C. Jeyabharathi (University of Greifswald, Germany) for the useful discussion.

\section{References}

1. Dincer, I.; Acar, C.; Int. J. Hydrogen Energy 2015, 40, 11094.

2. Chiesa, P.; Consonni, S.; Kreutz, T.; Williams, R.; Int. J. Hydrogen Energy 2005, 30, 747.

3. Poizot, P.; Dolhem, F.; Energy Environ. Sci. 2011, 4, 2003.

4. Lu, Q.; Hutchings, G. S.; Yu, W.; Zhou, Y.; Forest, R. V.; Tao, R.; Rosen, J.; Yonemoto, B. T.; Cao, Z.; Zheng, H.; Xiao, J. Q.; Chen, J. G.; Nat. Commun. 2015, 6, 6567.

5. Fan, X.; Zhou, H.; Guo, X.; ACS Nano 2015, 9, 5125.

6. Turner, J. A.; Science 2004, 305, 972.

7. Yan, W.; Hoekman, S. K.; Environ. Prog. Sustainable Energy 2014, 33, 213.

8. Khaselev, O.; Turner, J. A.; Science 1998, 280, 425.

9. Kiani, A.; Hatami, S.; Int. J. Hydrogen Energy 2010, 35, 5202. 
10. Pavel, C. C.; Cecconi, F.; Emiliani, C.; Santiccioli, S.; Scaffidi, S. A.; Caranorchi, S.; Comotti, M.; Angew. Chem., Int. Ed. 2014, 126, 1402 .

11. Li, J.; Zhou, X.; Xia, Z.; Zhang, Z.; Li, J.; Ma, Y.; Qu, Y.; J. Mater. Chem. A 2015, 3, 13066.

12. Fei, H.; Yang, Y.; Peng, Z.; Ruan, G.; Zhong, Q.; Li, L.; Samuel, E. L. G.; Tour, J. M.; ACS Appl. Mater. Interfaces 2015, 7, 8083.

13. Zou, X.; Huang, X.; Goswami, A.; Silva, R.; Sathe, B. R.; Mikmekova, E.; Asefa, T.; Angew. Chem., Int. Ed. 2014, 126, 4461.

14. Gong, M.; Wang, D. Y.; Chen, C. C.; Hwang, B. J.; Dai, H.; Nano Res. 2016, 9, 28.

15. Liu, X.; Cui, S.; Sun, Z.; Du, P.; Chem. Commun. 2015, 51, 12954.

16. Du, J.; Wang, J.; Ji, L.; Xu, X.; Chen, Z.; ACS Appl. Mater. Interfaces 2016, 8, 30205.

17. Zhao, J.; Tran, P. D.; Chen, Y.; Loo, J. S. C.; Barber, J.; Xu, Z. J.; ACS Catal. 2015, 5, 4115.

18. Raoof, J. B.; Ojiani, R.; Esfeden, S. A.; Nadimi, S. R.; Int. J. Hydrogen Energy 2010, 35, 3937.

19. Fan, F.; Peng, Z.; Ye, R.; Zhou, H.; Guo, X.; ACS Nano 2015 , 9, 7407.

20. Duan, J.; Chen, S.; Jaroniec, M.; Qiao, S. Z.; ACS Nano 2015, 9, 931

21. Yan, Y.; Xia, B.; Xu, Z.; Wang, X.; ACS Catal. 2014, 4, 1693.

22. Xiao, P.; Chen, W.; Wang, X.; Adv. Energy Mater. 2015, 5, 1500985.

23. Zhou, W.; Jia, J.; Lu, J.; Yang, L.; Hou, D.; Li, G.; Chen, S.; Nano Energy 2016, 28, 29.

24. Zou, X.; Zhang, Y.; Chem. Soc. Rev. 2015, 44, 5148.

25. Jia, F.; Yu, C.; Ai, Z.; Zhang, L.; Chem. Mater. 2007, 19, 3648.

26. Ge, X.; Wang, R.; Liu, P.; Ding, Y.; Chem. Mater. 2007, 19, 5827.

27. Li, Y.; Song, Y. Y.; Yang, C.; Xia, X. H.; Electrochem. Commun. 2007, 9, 981.
28. Jeong, H.; Kim, J.; Appl. Surf. Sci. 2014, $297,84$.

29. Chialvo, A. C.; Triaca, W. E.; Aravia, A. J.; J. Electroanal. Chem. 1984, 171, 303.

30. Nishio, K.; Masuda, H.; Angew. Chem., Int. Ed. 2011, 50, 1603.

31. Deng, Y.; Huang, W.; Chen, X.; Li, Z.; Electrochem. Commun. 2008, 10, 810 .

32. Zeis, R.; Lei, T.; Sieradzki, K.; Snyder, J.; Erlebacher, J.; J. Catal. 2008, 253, 132.

33. Jia, J.; Cao, L.; Wang, Z.; Langmuir 2008, 24, 5932.

34. Esakki Karthik, P.; Alagar Raja, K.; Senthil Kumar, S.; Phani, K. L. N.; Liu, Y.; Guo, Y. X.; Zhang, J.; Bond, A. M.; RSC Adv. 2015, 5, 3196.

35. Sai Sai Siddhardha, R. S.; Lakshminarayanan, V.; Ramamurthy, S. S.; J. Power Sources 2015, 288, 441.

36. Perez, J.; Gonzalez, E. R.; Villullas, H. M.; J. Phys. Chem. B 1998, 102, 10931.

37. Plowman, B.; Ippolito, S. J.; Bansal, V.; Sabri, Y. M.; Mullane, A. P.; Bhargava, S. K.; Chem. Commun. 2009, 5039.

38. Renjith, A.; Roy, A.; Lakshminarayanan, V.; J. Colloid Interface Sci. 2014, 426, 270.

39. Xiao, X.; Engelbrekt, C.; Li, Z.; Si, P.; Electrochim. Acta 2015 , $173,393$.

40. Sukeri, A.; Saravia, L. P. H.; Bertotti, M.; Phys. Chem. Chem. Phys. 2015, 17, 28510.

41. Sukeri, A.; Lima, A. S.; Bertotti, M.; Microchem. J. 2017, 133, 149.

42. Jeyabharathi, C.; Ahrens, P.; Hasse, U.; Scholz, F.; J. Solid State Electrochem. 2016, 20, 3025.

43. Ma, Y.-Y.; Wu, C.-X.; Feng, X.-J.; Tan, H.-Q.; Yan, L.-K.; Liu, Y.; Kang, Z.-H.; Wang, E.-B.; Li, Y.-G.; Energy Environ. Sci. 2017, 10, 788 . 